\title{
Resistance to rifampicin and isoniazid in strains of Mycobacterium tuberculosis
}

\author{
SALMAN H SIDDIQI, ${ }^{* 1}$ ABDUL AZIZ, $†$ ZULEMA REGGIARDO,$\ddagger^{2}$ \\ GARDNER MIDDLEBROOK $\ddagger^{2}$
}

From the *Pakistan Medical Research Council, Tuberculosis Research Center, Institute of Tuberculosis and Chest Diseases, †King Edward Medical College, Mayo Hospital, Lahore, Pakistan, and the $\ddagger$ Department

of Pathology, School of Medicine, University of Maryland, Baltimore, Maryland 21201

SUMMARY Drug susceptibility studies on strains of Mycobacterium tuberculosis isolated from widely different populations of patients and tested by two different techniques indicated that all 55 strains resistant to rifampicin were also resistant to isoniazid, while many strains resistant to isoniazid were found to be susceptible to rifampicin. This observation, which has as yet unknown laboratory and clinical significance, may be particularly useful in management of patients. Further studies are called for to establish this relation.

Rifampicin is a recent addition among antituberculous drugs. It is considered to be the most valuable and effective chemotherapeutic agent after isoniazid and is now widely used in the first line of antituberculous drugs.

Since the discovery and characterisation of this drug much work has been done on its bactericidal activity, mechanism of action, and development of resistance. ${ }^{1-4}$ It was noted that rifampicin, having a different chemical structure from isoniazid, has independent bactericidal action, and in wild strains of $M$ tuberculosis, the incidence of rifampicinresistant mutants was appreciably lower, while the serum level attained on standard oral dosage was much higher than isoniazid. ${ }^{5}$ Moreover, the pattern of resistance development to rifampicin was reported to be a single step type without having any intermediate level of resistance. ${ }^{35}$ Hobby (1968) compared the two drugs, isoniazid and rifampicin in vivo and in vitro and reported that on a weight basis rifampicin was approximately half as active as isoniazid against isoniazid-susceptible strains of $M$ tuberculosis. ${ }^{1}$ It has been reported that there is no antagonism between rifampicin and isoniazid, streptomycin, or ethambutol; and an absence of synergism between rifampicin and isoniazid has also been reported. ${ }^{2} 6$

\footnotetext{
'Present address: Johnston Laboratories, Cockeysville, Maryland 21030, USA.

2Present address: Department of Medical Microbiology, University of California, School of Medicine, Davis, California 95616, USA.

Accepted for publication 28 January 1981
}

There is little information concerning the properties of rifampicin-resistant strains. Hobby et al. ${ }^{2}$ working with the H37Rv strain mentioned that "cells resistant to rifampin in vitro appeared to be fully susceptible to other antimicrobial agents." Published reports, in general, have made casual mention of the absence of cross resistance between rifampicin and isoniazid without actually presenting supporting data. ${ }^{7}$

The purpose of this report is to present our observations on rifampicin resistance among strains of $M$ tuberculosis from two different studies in which all rifampicin-resistant strains were found to be isoniazid-resistant as well.

\section{Material and methods}

The subjects of the first study were tuberculous patients in Pakistan. The study included hospitalised patients as well as cases from outpatient clinics. The majority of these cases were chronic, while some were newly diagnosed patients with no history of previous treatment. The patients were tested at the Pakistan Medical Research Council's Tuberculosis Research Center, Mayo Hospital, Lahore.

A total of 1063 strains from the same number of patients, after identification as niacin-positive $M$ tuberculosis, were subjected to drug susceptibility testing by the standard proportion method using Löwenstein-Jensen (LJ) medium. ${ }^{8}$ The strains were tested against drugs listed in Table 1.

The critical proportion for resistance-that is, the percentage of resistant bacteria among a test 
Table 1 Selection of drugs and their concentrations for susceptibility testing

\begin{tabular}{lc}
\hline Drug & Concentration $(\mu \mathrm{g} / \mathrm{ml}$ LJ medium) \\
\hline Isoniazid (INH) & $0 \cdot 2$ \\
Streptomycin (SM) & $4 \cdot 0$ \\
p-aminosalicylic acid (PAS) & $0 \cdot 5$ \\
Ethionamide (ETA) & $20 \cdot 0$ \\
Ethambutol (EMB) & $2 \cdot 0$ \\
Thiacetazone (THA) & $2 \cdot 0$ \\
Rifampicin (RMP) & $40 \cdot 0$ \\
\hline
\end{tabular}

population beyond which the strain is considered resistant, was $1 \%$ for INH, RMP, PAS and EMB while for SM, ETA, and THA it was $10 \%$.

In another study, drug susceptibility was tested by an entirely different radiometric method in which ${ }^{14} \mathrm{CO}_{2}$ was measured as an index of growth in $7 \mathrm{H} 12$ medium containing $1 \mu \mathrm{Ci} / \mathrm{ml} 1-{ }^{14} \mathrm{C}$-palmitic acid. ${ }^{9} \mathrm{~A}$ total of $\mathbf{3 0 0}$ isolates of $M$ tuberculosis were provided by the Center for Disease Control (CDC), Atlanta. Drug susceptibility tests were done by the conventional plate method at CDC while the rapid radiometric method was employed at the University of Maryland. These cultures were tested against isoniazid, rifampicin, streptomycin, PAS and ethambutol.

\section{Results}

Among 1063 patients who were studied in Pakistan, 200 cases gave no history of previous treatment and all the isolated strains from these patients were found to be susceptible to rifampicin $(40 \mu \mathrm{g} / \mathrm{ml}$ in LJ medium), although isoniazid resistance was not infrequent among these cases. Among 863 chronic cases (mostly long treated), 21 were found to be excreting populations resistant to rifampicin. It was noted that all 21 of these strains were also resistant to isoniazid $(0.2 \mu \mathrm{g} / \mathrm{ml} \mathrm{LJ}$ medium), although not uniformly resistant to any other drug tested (see Table 2). Only six of 13 whose previous drug treatment was known had histories of treatment with rifampicin.

In the CDC study, 34 strains were found to be resistant to rifampicin $(1 \mu \mathrm{g} / \mathrm{ml}$ of $7 \mathrm{H} 12$ medium). All these were found to be resistant to isoniazid as well $(0.2 \mu \mathrm{g} / \mathrm{ml}$ of $7 \mathrm{H} 12$ medium). There was $95 \%$ agreement of these results with drug testing done independently on 7H10 medium on the same cultures at the Center for Disease Control.

\section{Discussion}

Rifampicin has been introduced only recently in Pakistan, and, due to its high cost, is not widely used, least of all among poor patients. Out of 21 rifampicinresistant cases, only six had a history of treatment
Table 2 Analysis of cases with rifampicin resistance

\begin{tabular}{|c|c|c|}
\hline No & $\begin{array}{l}\text { History of previous } \\
\text { treatment }\end{array}$ & Resistance to other drugs \\
\hline 1 & INH SM PAS EMB PZA & INH SM \\
\hline 2 & INH SM PAS EMB PZA & INH SM EMB \\
\hline 3 & $\begin{array}{l}\text { INH SM PAS EMB ETA } \\
\text { RMP }\end{array}$ & INH SM ETA EMB \\
\hline 4 & $\begin{array}{l}\text { INH SM PAS EMB THA } \\
\text { PZA RMP }\end{array}$ & INH SM EMB \\
\hline 5 & INH SM PAS THA PZA & INH SM PAS \\
\hline 6 & Chronic treated case & INH SM PAS ETA EMB \\
\hline 7 & Chronic treated case & INH THA \\
\hline 8 & INH SM PAS & INH \\
\hline 9 & Chronic treated case & INH SM PAS \\
\hline 10 & Treatment status not known & INH SM \\
\hline 11 & $\begin{array}{l}\text { INH SM PAS ETA EMB } \\
\text { THA PZA PMP }\end{array}$ & INH SM ETA \\
\hline 12 & Chronic treated case & INH SM \\
\hline 13 & $\begin{array}{l}\text { INH SM PAS ETA EMB } \\
\text { THA CYCL PYZ RMP }\end{array}$ & INH SM PAS EMB \\
\hline 14 & Chronic treated case & INH SM EMB \\
\hline 15 & Chronic treated case & INH SM \\
\hline 16 & INH SM THA & INH \\
\hline 17 & Chronic treated case & INH \\
\hline 18 & $\begin{array}{l}\text { INH SM PAS ETA EMB } \\
\text { RMP }\end{array}$ & INH SM EMB \\
\hline 19 & INH SM & INH \\
\hline 20 & INH EMB PZA RMP & INH SM \\
\hline 21 & INH SM PAS THA & INH SM PAS THA \\
\hline
\end{tabular}

INH $=$ isoniazid, $\mathbf{S M}=$ streptomycin, ETA $=$ ethionamide, EMB $=\infty$ ethambutol, THA $=$ thiacetazone, $\mathbf{R M P}=$ rifampicin, $\mathbf{P Z A}=$ pyra - zinamide, $\mathrm{CYCL}=$ cycloserine, $\mathrm{PAS}=p$-aminosalicylic acid.

with rifampicin. It was interesting to note that all these rifampicin-resistant cases had had various $\stackrel{2}{\circ}$ combinations of other antituberculous drugs and $\stackrel{\AA}{\complement}$ had developed resistance against other drugs as $\vec{\overrightarrow{ }}$ well, but apart from isoniazid, there was no con- 3 sistent pattern of resistance against any other drug.

The second observation was drawn out of an? altogether independent study concerned with development of a radiometric method for drug susceptibility? determination. The cultures were from patients' sputa which were submitted from all over the United States. Since this was a comparative study of two different methods of drug susceptibility testing, no information is available concerning the past history of treatment of these patients. However, it is $\frac{7}{2}$ interesting to note that all the 34 rifampicin strains were consistently resistant to isoniazid while this $N$ consistency was not observed in the case of any other drug.

In any event, with two different methods of testing for two quite different patient populations, it $\stackrel{0}{<}$ was interesting to find all rifampicin-resistant cases $\stackrel{0}{\varrho}$ also to beyielding strains resistant to $0 \cdot 2 \mu \mathrm{g}$ isoniazid/ $\Phi$ $\mathrm{ml}$ medium. This observation has some clinical $\stackrel{+}{-}$ significance. It would be interesting to investigate ${ }^{0}$ whether simple prolonged antituberculous treatment $\frac{\vec{P}}{\mathrm{D}}$ and emergence of resistance against other drugs are $\frac{\rho}{\square}$ some of the predisposing factors in the emergence of $\mathbb{\Phi}$ spontaneous rifampicin resistance. Selection of 
rifampicin-resistant mutant strains of $M$ tuberculosis in vitro does not result in simultaneous mutation to isoniazid resistance: the rifampicin $(250 \mu \mathrm{g} / \mathrm{ml})$ resistant mutant of $\mathrm{H} 37 \mathrm{Rv}$ from the Trudeau Mycobacterial Collection (No 331) is susceptible to $0 \cdot 2 \mu \mathrm{g}$ isoniazid/ml of $7 \mathrm{H} 12$ medium. Nevertheless, whatever the mechanism or mechanisms involved, it would appear that resistance to rifampicin in vivo usually emerges along with resistance to isoniazid and that the rifampicin resistance seems to arise after multiple drug treatment in which rifampicin itself was not necessarily included; the association of resistance against both of the most effective drugs used in tuberculosis treatment has very important implications for the management of patients. It should be pointed out that strains in these two studies were not tested in a medium containing both drugs together (to find out what proportion of the bacterial population was resistant to the drug combination).

Finally, it has been reported that a rifampicinresistant mutant strain, selected in vitro, was markedly less virulent for guinea pigs than the parent rifampicin-susceptible strain. ${ }^{10}$ No note was made of the isoniazid susceptibility or resistance or the catalase activity of this strain. Since rifampicin is such a valuable new drug, it would be worthwhile to investigate further the associative features of rifampicin and other antimycobacterial drugs.

\section{References}

${ }^{1}$ Hobby GL, Lenert TF. The antimycobacterial activity of rifampin. Am Rev Respir Dis 1968;97:713-4.

${ }^{2}$ Hobby GL, Lenert TF, Maier-Engallena J. In vitro activity of rifampin against the H37Rv strain of Mycobacterium tuberculosis. Am Rev Respir Dis 1969;99:45366.

${ }^{3}$ Tsukamura M. The pattern of resistance development to rifampicin in Mycobacterium tuberculosis. Tubercle 1972; 53:111-7.

4 Verbist L, Gyselen A. Antituberculous activity of rifampin in vitro and in vivo and the concentrations attained in human blood. Am Rev Respir Dis 1968;98:923-32.

${ }^{5}$ Canetti G, Le Lirzin M, Porven G, et al. Some comparative aspects of rifampicin and isoniazid. Tubercle 1968;49: 367-76.

- Dickinson JM, Mitchinson DA. Bactericidal activity in vitro and in guinea pig of isoniazid, rifampicin and ethambutol. Tubercle 1976;57:251-8.

${ }^{7}$ Lester W. Rifampin: a semisynthetic serivative of rifamycin-a prototype for the future. Annu Rev Microbiol 1972; 26:85-102.

${ }^{8}$ Canetti G, Fox W, Khomenko A, et al. Advances in techniques of testing mycobacterial drug sensitivity and the use of sensitivity tests in tuberculosis control programme. Bulletin World Health Organization 1969; 41:21-43.

${ }^{9}$ Middlebrook G, Reggiardo Z, Tigertt W. Automatable radiometric detection of growth of Mycobacterium tuberculosis in selective media. Am Rev Respir Dis 1977; 115:1066-9.

10 Ohsato T. Virulence of rifampicin resistant tubercle bacilli to experimental animals. A preliminary report. Kekkaku 1972;47:129-31.

Requests for reprints to: Dr SH Siddiqi, Johnston Laboratories, Cockeysville, Maryland 21030, USA. 